\title{
Analyse des chinesischen Pharmamarktes hinsichtlich der Produkte eines pharmazeutischen Unternehmens und Ansatzpunkte zur Markterschließung in China
}

\author{
Anja Preißler
}

In meiner Diplomarbeit zum Thema „Analyse des chinesischen Pharmamarktes hinsichtlich der Produkte eines pharmazeutischen Unternehmens und Ansatzpunkte zur Markterschließung in China" setzte ich mich mit den Besonderheiten der Geschäftstätigkeit eines mittelständischen Unternehmens in China auseinander.

Chinas Volkswirtschaft wies bereits in den achtziger Jahren wiederholt die weltweit höchsten Wachstumsraten auf, gleichzeitig ist das Land durch diesen Aufschwung zu einer "Lokomotive“ des pazifischen Wirtschaftsraumes geworden, die andere asiatische Länder wirtschaftlich "hinterherzieht". Die USA, Japan und Europa werden ihre bisherige starke Position im Welthandel in Zukunft gegen die wirtschaftlich expandierenden asiatischen Staaten behaupten müssen.

Mittelständische Unternehmen sind in den asiatischen Märkten, vor allem wegen deren schwieriger Markterschließung, noch selten vertreten. Doch gerade ihnen kommt in der exportorientierten Wirtschaft Deutschlands eine Schlüsselstellung zu.

In meiner Diplomarbeit beschreibe ich den Weg eines mittelständischen Pharmaunternehmens auf den chinesischen Markt, schildere Chancen in China als auch Risiken. Dazu habe ich ein Marketingkonzept für die strategische Markterschließung erarbeitet. Das überproportionale Wachstum des chinesischen Pharmamarktes eröffnet einem Unternehmen, das daran partizipieren möchte, heute große Chancen, erfordert aber auch ein entschlossenes Vorgehen.

Die Berlin-Chemie AG, die auf internationaler Ebene bisher vor allem in Osteuropa erfolgreich ihre Produkte verkauft, strebt eine Erschließung dieses vielversprechenden Marktes an. Dieser Aspekt bildet den Ausgangspunkt des Konzepts, dessen Aussagen das Unternehmen auf dem Weg in das Reich der Mitte unterstïtzen und zum Erfolg des Auslandsgeschäftes beitragen sollen.

Nachfolgend möchte ich meine Vorgehensweise und einige Schwierigkeiten, die bei der Ausarbeitung auftraten, schildern.

Zunächst ermöglichte mir die Verwendung von Instrumenten und Methoden des Internationalen Marketings und Managements eine genaue Analyse des Marktes und des Unternehmensumfeldes.

Der erste Teil der Arbeit befaßt sich mit der umfassenden Analyse des chinesischen Pharmamarktes hinsichtlich des Gesundheitswesens und des Marktumfelds der dort tätigen pharmazeutischen Unternehmen. Er gibt u. a. einen Überblick zur Entwicklung bestimmter Indi- kationen, zum Arzneimittelkonsum in China, dem Therapieverhalten chinesischer Ärzte und den Marktzugangsbedingungen, mit denen ausländische Unternehmen in China konfrontiert werden. Hierzu wurden Daten statistischer Erhebungen genutzt sowie Veröffentlichungen von Fachzeitschriften und Datenbanken zu Rate gezogen.

Die Aufbereitung der so gewonnenen Daten ist die Basis für Ableitungen, die sich für einen erfolgreichen Markteintritt der Berlin-Chemie AG ergeben und die im nachfolgenden zweiten Hauptteil behandelt werden. Doch zunächst erfolgt die Formulierung der unternehmerischen Zielsetzung für den chinesischen Markt und eine ausführliche Stärken-Schwächen-Analyse unter Beachtung der Einflußfaktoren des Unternehmensumfeldes. Das Ergebnis des zweiten Teils der Arbeit sind Handlungsempfehlungen für das Unternehmen in Form einer möglichen Internationalisierungsstrategie und eines Marketingmix für den chinesischen Markt.

Die Informationsbeschaffung ist eine der schwierigsten Aufgaben einer Marktanalyse, denn es gilt, in der Fülle der heute verfuigbaren Informationen eine aussagefähige und effiziente Datenerhebung durchzufuihren. Was den chinesischen Markt betrifft, so sind Daten durch Datenbanken noch wenig standardisiert und wenn, dann teuer abrufbar. Deshalb entstammen einige der in der Arbeit verwendeten Daten älteren Erhebungen und Publikationen und wurden von mir, so gut es ging, auf den heutigen Stand bezogen.

Einen Großteil der Informationen konnte ich im Unternehmen vorfinden und auch auswerten, da Berlin-Chemie schon seit längerer Zeit den Markteintritt plante und entsprechende Vorbereitungen traf. Andere und oftmals aktuellere Informationen und Daten gewann ich bei Recherchen im Internet und in Wirtschaftszeitschriften wie der „China Business Review“, dem „Economist“ und in speziellen Pharma-Publikationen.

Interessiert verfolgte ich die damalige und auch noch derzeit aktuelle Diskussion zum Thema „Joint-Venture versus Tochterunternehmen in Asien " in diesen Medien, in denen Vor- und Nachteile der jeweiligen Alternative aufgezeigt wurden.

Diese Kontroverse übernahm ich in meine Arbeit und untersuchte sie im Kapitel „Internationalisierungsstrategie" im Hinblick auf das Profil der Niederlassung der Berlin-Chemie in China.

Die Berlin-Chemie AG hatte bereits Erfahrung mit einem chinesischen Kooperationspartner gemacht. Die Partnerschaft mit einem chinesischen Unternehmen sollte 
dem Aufbau eines Vertriebsnetzes in China und der Plazierung der Berlin-Chemie Produkte auf dem chinesischen Markt dienen. Nach anfänglich guten Umsätzen verschlechterte sich die Zusammenarbeit beider Partner aufgrund von Vertragsstreitigkeiten und Verständnisschwierigkeiten, so daß die Kooperation schließlich nicht fortgeführt wurde und das Unternehmen heute zielgerichtet eine eigene Marktpräsenz aufbaut.

Der Ländermarkt China stand 1997 an 10. Stelle unter den Exportmärkten der Berlin-Chemie (nach den Hauptumsatz-Märkten Russland, GUS-Länder, Polen, Bulgarien usw.) und gewinnt zunehmend an Bedeutung. Beeindruckend ist dieses Potential, wenn man bedenkt, daß diese Position trotz eines bisher nur kleinen und unvollständigen Sortiments in China und ohne die üblichen Werbemaßnahmen erreicht wurde - im Vergleich zu den gut erschlossenen osteuropäischen Märkten.

Die von mir erarbeitete Marketingstrategie zielt auf die Besonderheiten des Einsatzes der Marketinginstrumente in China. Produkt-, Kommunikations-, Preis- und Distributionspolitik unterscheiden sich grundlegend von in Europa praktiziertem Marketing.

Gesetzliche Regulierungen und Vorschriften beeinflussen die Preisbildung von Produkten und die Werbung für ausländische Produkte in den chinesischen Medien. Eine völlig andere Distributions- und Vertriebsstruktur in China erfordert - eröffnet aber auch - neue und andere Möglichkeiten des Vertriebs und beispielsweise des Direktmarketings.

Aufgrund der dynamischen Entwicklung der chinesischen Märkte ist es erforderlich, daß die dort tätigen Unternehmen ihre Marketingpolitik und Strategien einem ständigen Anpassungs- und Überarbeitungsprozeß unterziehen. Die gesetzlichen Rahmenbedingungen und die wirtschaftlichen Entwicklungen verändern sich mitunter sehr schnell. Was heute noch aktuell ist, kann morgen schon überholt sein. Deshalb ist es für Unternehmen, die in China tätig werden wollen, empfehlenswert, einen Mitarbeiter zu definieren, der für den Ländermarkt China zuständig und über aktuelle Vorgänge informiert ist.

Letztlich bin ich der Auffassung, daß eine Marktanalyse und die Erarbeitung einer Strategie zu den Grundvoraussetzungen gehört, bevor ein ausländischer Markt betreten wird.

Die Methoden und Instrumente des Internationalen Marketings ermöglichen die Entscheidungen und Planungen des Auslandsgeschäfts und sind deshalb auf Unternehmensebene unentbehrlich geworden.

Die in der Diplomarbeit dargelegten Empfehlungen zu Markterschließung und Marketingstrategie sollen die strategische Planung des China-Geschäfts der BerlinChemie AG erleichtern helfen und auch inspirieren. Dem Leser soll diese Arbeit einen ersten Eindruck von der chinesischen Pharmabranche vermitteln sowie Anregungen geben, auf welche Art ein Unternehmen ganz gleich für welche Art von Produkten - den chinesischen Markt für sich erschließen kann.

Zusammenfassend läßt sich nach meiner Ansicht folgende Aussage treffen: China, ja der gesamte asiatisch-pa- zifische Raum ist kein einfacher Markt, er ist für westliche Firmen Chance und Bedrohung in einem. Die Bedrohung, das waren bis vor kurzem Asiens Exporte zu unschlagbaren Preisen. Und dennoch: „In der Region nicht präsente Unternehmen merken oft gar nicht, welche Umsatz-, Gewinn- oder Lernchancen ihnen entgehen - und daß ihre Abwesenheit die größte Gefahr für sie birgt: die langfristige Minderung der Wettbewerbsfähigkeit. Unternehmen, die in einer dynamischen Wachstumsregion operieren, akkumulieren, bei sonst gleichen Umständen, komparative Vorteile gegenüber denen in stagnierenden Märkten.

\section{Verfasserin}

\section{Anja Preißler}

(4. Studienjahr, Betriebswirtschaftslehre)

Technische Fachhochschule Wildau

Bahnhofstraße

15745 Wildau 